\title{
Non-radioactive Assay to Determine Product Profile of Short-chain Isoprenyl Diphosphate Synthases
}

\author{
Avanish Rai and Dinesh A. Nagegowda*
}

\begin{abstract}
Molecular Plant Biology and Biotechnology Lab, CSIR-Central Institute of Medicinal and Aromatic Plants, Research Centre, Bengaluru - 560065, India

*For correspondence: da.nagegowda@cimap.res.in
\end{abstract}

\begin{abstract}
[Abstract] Isoprenoids represent the largest class of metabolites with amazing diversities in structure and function. They are involved in protecting plants against pathogens or herbivores or involved in attracting pollinators. Isoprenoids are derived from geranyl diphosphate (GPP; $\mathrm{C}_{10}$ ), farnesyl diphosphate (FPP; $\mathrm{C}_{15}$ ), geranylgeranyl diphosphate (GGPP; $\mathrm{C}_{20}$ ), and geranylfarnesyl diphosphate (GFPP; $\mathrm{C}_{25}$ ) that are in turn formed by sequential condensations of isopentenyl diphosphate (IPP; $\mathrm{C}_{5}$ ) with an allylic acceptor such as dimethylallyl diphosphate (DMAPP; C $_{5}$ ), GPP, FPP, or GGPP in a reaction catalyzed by isoprenyl diphosphate synthases (IDSs). IDS enzyme assay for determination of prenyl diphosphate products is generally performed using radiolabelled substrates, and the products formed are identified by employing expensive instruments such as phosphor imager, radio-GC, or radioHPLC. Though a non-radioactive assay for measuring IDS activity in crude plant extract has been reported, it requires a complex methodology utilizing chromatography coupled with tandem mass spectrometry (LC/MS-MS). Here, we describe a non-radioactive and simple inexpensive assay for determining the IDS assay products using non-radiolabeled IPP and its co-allylic substrates DMAPP, GPP, and FPP. The detection of prenyl diphosphate products generated in the assay was highly efficient and spots corresponding to prenyl alcohols were visible at $>40 \mu \mathrm{M}$ concentrations of IPP and DMAPP/GPP/FPP substrates. The protocol described here is sensitive, reliable, and technically simple, which could be used for functional characterization of IDS candidates.
\end{abstract}

Keywords: Isoprenyl diphosphate synthase, Non-radioactive, Phosphatase, Apyrase, Prenyl alcohol, Thin layer chromatography

[Background] Short-chain IDSs catalyze the chain length elongation reaction (1'-4 condensations of IPP units) in which allylic isoprenoids substrates $\mathrm{C}_{5}$-DMAPP, $\mathrm{C}_{10}$-GPP, or $\mathrm{C}_{15}$-FPP couple with IPP to generate linear isoprenoids (Figure 1). For instance, Catharanthus roseus GPPS large subunit (CrGPPS.LSU) and GGPP synthase 2 (CrGGPPS2) catalyze the formation of GPP/GGPP [Figure 4 in Rai et al., 2013] and GGPP [Figure 3 in Kumar et al., 2020], respectively, by sequential incorporation of IPP units with DMAPP, GPP, or FPP as substrates. The most widely used assay for determination of IDS activity is discontinuous, time and labor intensive, and involves the use of IPP radiolabeled with either ${ }^{14}[\mathrm{C}]$ or ${ }^{3}[\mathrm{H}]$. A non-radioactive and LC-MS-based method for determining IDS activity has been reported using crude plant extract utilizing chromatography coupled with tandem mass spectrometry (LC/MS-MS) (Nagel et al., 2012). The protocol described here provides an efficient approach to 
determine the IDS activity without the need for radioactive substrates and high-end instrumentation. The method involves thin layer chromatography (TLC) separation of IDS assay products and visualization and quantification by iodine staining. The assay products generated can be extracted from TLC plate and further verified through LC/MS-based approach as reported in Kumar et al., 2020. We have used purified CrGPPS.LSU, a bifunctional G(G)PP synthase (EC 2.5.1.29) catalyzing the formation of both GPP and GGPP (Rai et al., 2013) in this protocol. The IDS assay described here is highly specific, sensitive, and technically simple which can be useful in determining the functional activity of short-chain IDS enzymes. The efficiency of enzymatic product detection with this method is comparable to that of previously reported radioactive and non-radioactive assays in terms of substrate concentrations.

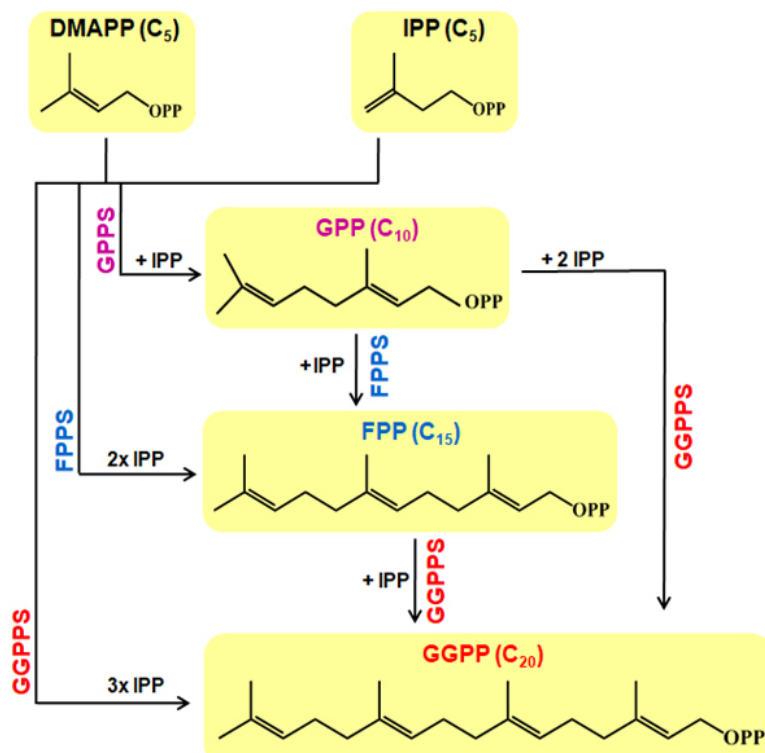

Figure 1. IPP and DM APP are utilized in the formation of GPP $\left(C_{10}\right), \operatorname{FPP}\left(C_{15}\right)$, and GGPP $\left(C_{20}\right)$ in a reaction catalyzed by short-chain IDS such as geranyl diphosphate synthases (GPPS), farnesyl diphosphate synthases (FPPS), and geranylgeranyl diphosphate synthase (GGPPS), respectively. While FPPS catalyzes the formation of FPP by condensation of DMAPP with 2 IPP units or GPP with one IPP unit, GGPPS forms of GGPP by condensation of DMAPP with three IPP units, GPP with two IPP units, or FPP with one IPP unit.

\section{Materials and Reagents}

Note: The materials and reagents not provided with company and catalog number can be ordered from any qualified company for using in this experiment.

1. Pipette tips (Axygen, USA)

2. $1.5 \mathrm{ml}$ Microcentrifuge tubes (Eppendorf, catalog number: T9661)

3. Parafilm (Sigma-Aldrich, catalog number: P7793)

4. Pencils

5. Disposable latex gloves 
6. Poly-Prep chromatography columns (Bio-Rad, catalog number: 7311550)

7. Disposable PD-10 desalting columns (GE Healthcare, catalog number: GE17-0851-01)

8. Purified isoprenyl diphosphate synthase [Catharanthus roseus GPPS large subunit (CrGPPS.LSU) for this protocol]

9. Rosetta 2(DE3) Competent Cells (Novagen, catalog number: 71400-M)

10. pET-28a(+) vector (Novagen, catalog number: 69864)

11. $\mathrm{Ni}^{2+}$-charged nitrilotriacetic acid (NTA) Agarose (Bio-Rad, catalog number: 7800800)

12. Luria Bertani Broth, Miller (HIMEDIA, catalog number: M1245)

13. Luria Bertani Agar (HIMEDIA, catalog number: M1151F)

14. Kanamycin sulfate (Sigma-Aldrich, catalog number: 10106801001)

15. Chloramphenicol (Sigma-Aldrich, catalog number: C0378)

16. Imidazole (Sigma-Aldrich, catalog number: I5513-25G)

17. Lysozyme from hen egg white (Sigma-Aldrich, catalog number: 10837059001)

18. Isopentenyl diphosphate (Echelon Biosciences, catalog number: I-0050)

19. Dimethylallyl diphosphate (Echelon Biosciences, catalog number: I-0051)

20. Geranyl diphosphate (Echelon Biosciences, catalog number: I-0100)

21. Farnesyl diphosphate (Echelon Biosciences, catalog number: I-0150)

22. Apyrase from potatoes (Sigma-Aldrich, catalog number: A6132)

23. Alkaline phosphatase from calf intestine (CIP) (Sigma-Aldrich, catalog number: P4978)

24. Geraniol (Sigma-Aldrich, catalog number: 48798)

25. Farnesol (Sigma-Aldrich, catalog number: 43348)

26. Geranylgeraniol (Sigma-Aldrich, catalog number: G3278)

27. TLC Silica gel 60 RP-18 F254S (Merck, catalog number: 105560)

28. Glycine (Sigma-Aldrich, catalog number: G7126-100G)

29. $10 \%$ Mini-PROTEAN ${ }^{\circledR}$ TGX $^{\mathrm{TM}}$ precast protein gels (Bio-Rad, catalog number: 4561033)

30. Sodium dodecyl sulfate (SDS) (Sigma-Aldrich, catalog number: L3771-100G)

31. Bradford Protein Assay Dye Reagent (Bio-Rad, catalog number: 5000006)

32. Coomassie brilliant blue (CBB-R-250) (Sigma-Aldrich, catalog number: B-7920-10G)

33. Bromophenol blue (Sigma-Aldrich, catalog number: B5525-5G)

34. Ethanol (Emsure, catalog number: 1.00983.0511)

35. Water HPLC grade (Sigma-Aldrich, catalog number: 270733)

36. Glycerol (Sigma-Aldrich, catalog number: G5516)

37. 3-Morpholino-2-hydroxypropanesulfonic acid (MOPSO) (Sigma-Aldrich, catalog number: M8389)

38. 1,4-Dithiothreitol (DTT) (Sigma-Aldrich, catalog number: 10197777001)

39. Magnesium chloride $\left(\mathrm{MgCl}_{2}\right)$ (HIMEDIA, catalog number: GRM4340)

40. lodine $\left(I_{2}\right)$ crystals (Sigma-Aldrich, catalog number: 229695)

41. $n$-Hexane (Merck, catalog number: 104369)

42. TRIS hydrochloride (Tris-HCl) (HIMEDIA, catalog number: MB030) 
43. Methanol (Merck, catalog number: 60600905001730)

44. Isopropyl-1-thio- $\beta$-D-galactopyranoside (IPTG) (Sigma-Aldrich, catalog number: 15502-5G)

45. Lysis buffer (see Recipes)

46. Wash buffer (see Recipes)

47. Elution buffer (see Recipes)

48. Storage buffer (see Recipes)

49. Assay buffer (see Recipes)

50. Dephosphorylation buffer (see Recipes)

51. Mobile phase (see Recipes)

52. TG buffer (see Recipes)

\section{Equipment}

Note: The equipment not provided with company and catalog number can be ordered from any qualified company for using in this experiment.

1. Pipettes

2. Eppendorf Thermo Mixer ${ }^{\circledR}$ F1.5 (Eppendorf, catalog number: 5384000012)

3. Glass rectangular developing chamber $20 \times 20 \mathrm{~cm}$ (with lid)

4. Measuring cylinders

5. Scale ruler

6. Tweezers

7. Scissors

8. Borosilicate beakers, capacity $500 \mathrm{ml}$

9. Rocker (GeNei, catalog number: 107106GB)

10. Variable volume pipettes (Eppendorf)

11. Magnetic stirrer (GeNei, catalog number: 117795GB)

12. Adsorbent TLC scraper (Sigma-Aldrich, catalog number: Z265268)

13. Vacuum concentrator (Savant Speed Vac, catalog number: SPD131DDA)

14. Fume hood

15. Sonicator (PRO Scientific, catalog number: $\mathrm{H}-1021-2$ )

16. Circulating refrigerated water bath

17. ThermoMixer C (Eppendorf, catalog number: 5382000015)

18. Vortex mixer (GeNei, catalog number: 106887GB)

19. Centrifuge 5424 R (Eppendorf, catalog number: 5404000014)

20. Digital camera or scanner

\section{Software}

1. ImageJ (National Institutes of Health, USA, https://imagej.nih.gov/ij/) 


\section{Procedure}

A. Expression and purification of recombinant isoprenyl diphosphate synthase protein

1. Transform Escherichia coli rosetta-2 cells with plasmid harboring IDS of interest (Here, we have used pET28a:CrGPPS.LSU). The GeneBank loci IDs for nucleotide and protein sequences are JX417183 and AGL91645, respectively. For more information on enzyme characteristics of CrGPPS.LSU, please refer to Rai et al., 2013.

2. Inoculate a single colony in $25 \mathrm{ml}$ Luria-Bertani (LB) medium with $37 \mathrm{mg} / \mathrm{ml}$ chloramphenicol and $50 \mathrm{mg} / \mathrm{ml}$ kanamycin.

3. Grow overnight $(16 \mathrm{~h})$ at $37{ }^{\circ} \mathrm{C}$ with $200 \mathrm{rpm}$ and transfer $5 \mathrm{ml}$ of overnight grown culture to $1,000 \mathrm{ml}$ of LB medium with $37 \mathrm{mg} / \mathrm{ml}$ chloramphenicol and $50 \mathrm{mg} / \mathrm{ml}$ kanamycin.

4. Place the culture flask in an incubator shaker set at $37^{\circ} \mathrm{C}$ and $200 \mathrm{rpm}$ and grow the cells until the $\mathrm{OD}_{600}$ reaches 0.5 .

5. Add isopropyl-1-thio- $\beta$-D-galactopyranoside (IPTG) to a final concentration of $0.4 \mathrm{mM}$ and continue growing cultures for an additional $18 \mathrm{~h}$ at $18^{\circ} \mathrm{C}$.

6. Centrifuge cultures at $6,000 \times g$ for $10 \mathrm{~min}$ at $4{ }^{\circ} \mathrm{C}$ to obtain cell pellets.

7. Proceed to the next step or store the pellet at $-80^{\circ} \mathrm{C}$ until further use.

8. Resuspend cell pellet in lysis buffer with $2-5 \mathrm{ml}$ per gram cell pellet.

9. Add $1 \mathrm{mg} / \mathrm{ml}$ lysozyme to the cell suspension and keep on ice for $30 \mathrm{~min}$.

10. Sonicate the cell suspension using a sonicator equipped with microtip and give six $10 \mathrm{~s}$ bursts with a $10 \mathrm{~s}$ cooling period.

11. Recover the lysate by centrifugation at $12,000 \times \mathrm{g}$ for 20 min at $4{ }^{\circ} \mathrm{C}$ and transfer to a $50 \mathrm{ml}$ tube.

12. Take $1 \mathrm{ml}$ of $50 \% \mathrm{Ni}^{2+}-\mathrm{NTA}$ agarose slurry and remove the storage solution by brief centrifugation at $1,000 \times g$ for $15 \mathrm{~s}$ and resuspend the $\mathrm{Ni}^{2+}$-NTA agarose in lysis buffer. Add 1 $\mathrm{ml}$ of resuspended $\mathrm{Ni}^{2+}$-NTA resin to the recovered lysate in the previous step and keep the tube on rotor shaker for $1 \mathrm{~h}$ at $4{ }^{\circ} \mathrm{C}$.

13. Transfer $\mathrm{Ni}^{2+}$-NTA resin (containing captured His-tagged protein) to the $0.8 \times 4 \mathrm{~cm}$ Poly-Prep chromatography column and allow to settle by gravity.

14. Equilibrate the column with 5 column volumes of lysis buffer containing $10 \mathrm{mM}$ Imidazole.

15. Wash the $\mathrm{Ni}^{2+}-\mathrm{NTA}$ resin with 5 column volumes of wash buffer containing $20 \mathrm{mM}$ Imidazole to remove the unbound protein.

16. Elute the bound protein and collect the fractions with $3 \mathrm{ml}$ of elution buffer containing $250 \mathrm{mM}$ Imidazole.

17. Equilibrate PD-10 desalting column with $4 \mathrm{ml}$ of storage solution and transfer protein eluant to the column.

18. Add $2 \mathrm{ml}$ storage buffer and collect eluant in $0.5 \mathrm{ml}$ fractions (E1-E4).

19. Load $30 \mu$ protein sample on $10 \%$ SDS gel and perform electrophoresis in Tris-Glycine (TG) buffer. 
20. Determine the concentration of purified protein using the Bradford method (Bradford, 1976).

B. Isoprenyl diphosphate synthase assay

1. Perform IDS assay by adding equimolar concentrations (10 $\mu \mathrm{M}$ to $80 \mu \mathrm{M})$ of IPP and DMAPP, IPP and GPP, and IPP and FPP in a final volume of $200 \mu$ of assay buffer in separate $1.5 \mathrm{ml}$ centrifuge tubes.

2. Add $5 \mu \mathrm{g}$ of purified IDS enzyme (CrGPPS.LSU in this assay) to each tube to initiate the reaction.

3. Take three separate $1.5 \mathrm{ml}$ tubes and add $5 \mu \mathrm{g}$ of heat inactivated ( $95{ }^{\circ} \mathrm{C}$ for $10 \mathrm{~min}$ ) CrGPPS.LSU for negative controls in a final volume of $200 \mu \mathrm{l}$ of assay buffer containing equimolar concentrations ( $40 \mu \mathrm{M})$ of IPP and DMAPP, IPP and GPP, and IPP and FPP.

4. Incubate all tubes containing IDS assay reaction mixture at $30^{\circ} \mathrm{C}$ in a circulating water bath or ThermoMixer for $6 \mathrm{~h}$.

5. Add $200 \mu \mathrm{l}$ of dephosphorylation buffer and continue to incubate for $16 \mathrm{~h}$ at $30{ }^{\circ} \mathrm{C}$ in a ThermoMixer to hydrolyze all diphosphate esters (unreacted substrates as well as prenyl products).

6. Add $1 \mathrm{ml} n$-hexane and vortex vigorously for $30 \mathrm{~s}$ to extract the hydrolyzed reaction products and substrates (prenyl alcohols).

7. Centrifuge for $30 \mathrm{~s}$ at $12,000 \times \mathrm{g}, 25^{\circ} \mathrm{C}$.

8. Carefully transfer the upper hexane fraction (approximately $800 \mu \mathrm{l}$ ) containing the prenyl alcohols to a new $1.5 \mathrm{ml}$ centrifuge tube using a $1 \mathrm{ml}$ pipette.

9. Concentrate the collected hexane fraction containing the prenyl alcohols to $25 \mu \mathrm{l}$ using a SpeedVac (at $30^{\circ} \mathrm{C}$ for $30 \mathrm{~min}$ ) and immediately use it for TLC analysis.

C. Determination of IDS product profile

1. Take a $500 \mathrm{ml}$ beaker and add mobile phase solvent to a depth of about $0.5 \mathrm{~cm}$. Seal the beaker with parafilm, swirl it gently and allow it to stand for 2 to $5 \mathrm{~min}$ for saturation of the chamber.

2. Take a reverse phase TLC silica gel plate with a dimension of $5.0 \times 7.5 \mathrm{~cm}$. Draw a horizontal line with a pencil, $1 \mathrm{~cm}$ above from both edges of the bottom.

3. Mark five spots along the line on TLC silica gel plates at equidistance (approximately 5-7 mm apart) for applying hexane fraction containing prenyl alcohols.

4. Prepare prenyl alcohol standard solution in hexane containing $40 \mu \mathrm{M}$ each of geraniol, farnesol, and geranylgeraniol.

5. Use a $10 \mu \mathrm{l}$ pipette to carefully spot the samples on the marked spots of TLC silica gel plate. After spotting each sample, blow gently on the TLC plate to evaporate the solvent.

a. Spot the standard solution containing $\mathrm{GOH}, \mathrm{FOH}$, and $\mathrm{GGOH}$ in lane S.

b. Spot $20 \mu \mathrm{l}$ of the concentrated hexane fraction of negative control at lane number 1 .

c. Spot $20 \mu \mathrm{l}$ hexane of the extracted prenyl alcohol products from different enzymatic reactions on the corresponding TLC plates (Figure 2). 
6. Use tweezers to place the prenyl alcohols spotted TLC silica gel plate in the beaker containing the mobile phase.

7. Perform TLC chromatographic separation by sealing the beaker with parafilm and allow solvent front to rise upward.

8. Take out the TLC silica gel plate from the beaker just before the solvent front reaches the top end (about $15 \mathrm{~min}$ ), mark the solvent front with a pencil and allow the TLC silica gel plate to dry for 5-10 min.

9. Stain the TLC plate by exposing it to iodine vapors for 3 to $5 \mathrm{~min}$ in a rectangular glass chamber containing iodine crystals (Figure 3b, Kumar et al., 2020).

10. Visualize the prenyl alcohol spots corresponding to product and substrates by comparing with reference standards (Rai et al., 2013).

11. Document the TLC image with prenyl alcohol spots using a digital camera or scanner.

12. The documented product spots can be relatively quantified using ImageJ software.

D. Verification of prenyl alcohol products

1. After documenting the TLC images, circle the spots and scrape the corresponding spots of prenyl diphosphate products parallel to the reference compounds using TLC scraper.

2. Elute the prenyl diphosphate product and reference compound in methanol.

3. Concentrate the eluted compounds under $\mathrm{N}_{2}$ gas stream.

4. Perform standard liquid chromatography mass spectrometry (LC-ESI-MS) according to the protocol described in Kumar et al. (2020) in a positive mode to confirm prenyl diphosphate products and to rule out any possible contamination from acid hydrolysis reactions (Figure 3c, Kumar et al., 2020).

\section{Data analysis}

In this methodology, a non-radioactive IDS assay has been employed to measure the activity of the CrGPPS.LSU which provides metabolic flux for both primary and specialized metabolites in $C$. roseus (Rai et al., 2013). CrGPPS.LSU has been well characterized and therefore, serves as an appropriate reference enzyme to validate the prenyl alcohol product profile generated using this non-radioactive assay. IDS assay was performed in the presence of CrGPPS.LSU and the formation and visualization of prenyl alcohols ( $\mathrm{GOH}$ and $\mathrm{GGOH}$ ) was carried out (Figure 2). The optimal conditions for the prenyl alcohol product identification were explored by probing the intensities of product spots arising after using different concentrations of substrates. The spots corresponding to $\mathrm{GOH}$, and GGOH prenyl alcohol products were visible at $\geq 20 \mu \mathrm{M}$ concentration of IPP and DMAPP substrates (Figure 2A). However, the spots corresponding to GGOH were detectable in a linear range and clearly visible at $\geq 10 \mu \mathrm{M}$ concentration of IPP and GPP substrates (Figure 2B) and $\geq 40$ $\mu \mathrm{M}$ concentration of IPP and FPP substrates (Figure $2 \mathrm{C}$ ). Therefore, the results from the nonradioactive IDS assay demonstrate that about $40-80 \mu \mathrm{M}$ of IPP and DMAPP/GPP/FPP substrate 
concentration was ideal to achieve the optimal and detectable level of prenyl alcohol product from the assay (Figure 2).

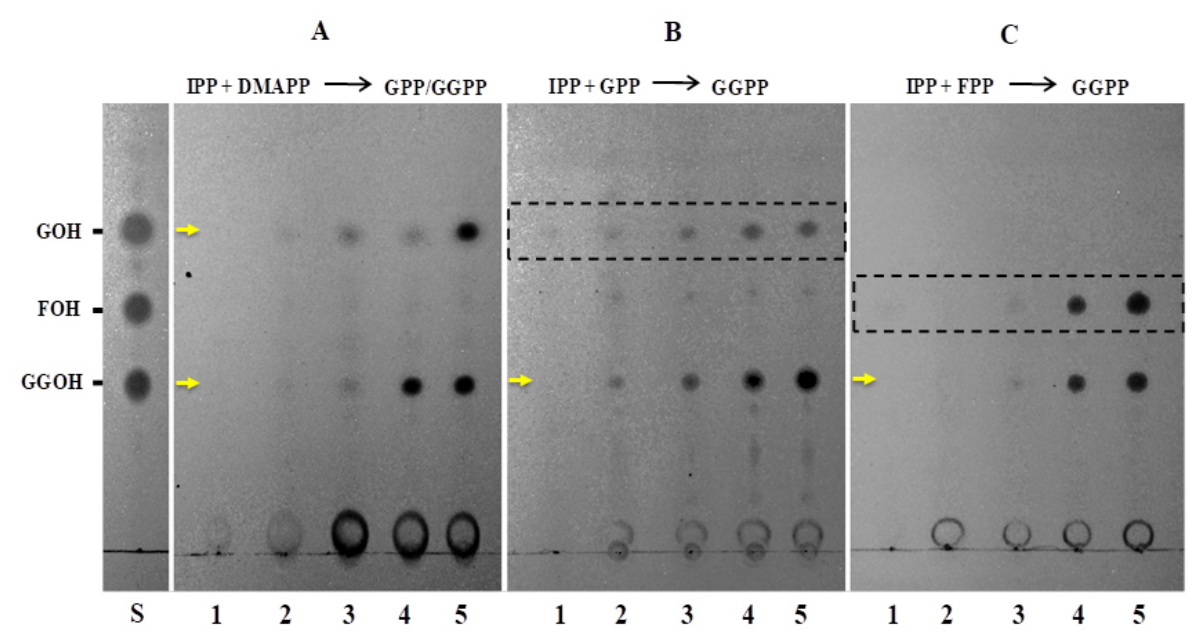

Figure 2. In vitro IDS assays of recombinant (His) ${ }_{6}$-CrGPPS.LSU. Spots on the TLC plates correspond to reaction products from CrGPPS.LSU assays using IPP and DMAPP (plate A), IPP and GPP (plate B), and IPP and FPP (plate C). Products formed are indicated by arrows and the spots in dashed boxes are unreacted substrates. The products were confirmed by comparing the spots with authentic geraniol $(\mathrm{GOH})$, farnesol $(\mathrm{FOH})$, and geranylgeraniol (GGOH) standards (lane S). Lane 1 in A, B, and C TLC plates represents negative control in which boiled CrGPPS.LSU protein was assayed with $40 \mu \mathrm{M}$ each of IPP and DMAPP, IPP and GPP, and IPP and FPP, respectively. Lanes 2-5 represent reaction products of CrGPPS.LSU protein assayed with $10 \mu \mathrm{M}, 20 \mu \mathrm{M}, 40 \mu \mathrm{M}$, and $80 \mu \mathrm{M}$ each of IPP and DMAPP (plate A), IPP and GPP (plate B), and IPP and FPP (plate C), respectively. Lane S: Authentic prenyl alcohol standard mix.

\section{Notes}

1. The standards geraniol, farnesol, and geranylgeraniol were not used as carriers in all chromatographic separations of prenyl alcohol products.

2. Overnight incubation at $30^{\circ} \mathrm{C}$ after adding dephosphorylation buffer is necessary to completely hydrolyze diphosphate esters.

3. To minimize background smear and for better chromatographic separation, carefully remove the hexane fraction, the organic layer (in the B8 step).

4. Wear gloves while handling the TLC plates contamination.

5. Sample spotting diameter should not be more than 3-4 $\mathrm{mm}$.

6. Mark the TLC plates very gently with a pencil to avoid damaging the silica gel.

7. The level of mobile phase should not cover spots when the TLC plate is placed in the beaker. 
8. Always use freshly prepared mobile phase solvents for better resolution of the spots and reproducibility of the results.

9. The product and substrate spots should be circled with a pencil upon removal of TLC plates from iodine vapor chamber as they fade away after a few minutes.

\section{$\underline{\text { Recipes }}$}

1. Lysis buffer (10 ml)

$50 \mathrm{mM} \mathrm{NaH}_{2} \mathrm{PO}_{4}$

$300 \mathrm{mM} \mathrm{NaCl}$

$10 \mathrm{mM}$ Imidazole, $\mathrm{pH}-8.0$

2. Wash buffer $(100 \mathrm{ml})$

$50 \mathrm{mM} \mathrm{NaH}_{2} \mathrm{PO}_{4}$

$300 \mathrm{mM} \mathrm{NaCl}$

$20 \mathrm{mM}$ Imidazole, $\mathrm{pH}-8.0$

3. Elution buffer ( $5 \mathrm{ml})$

$50 \mathrm{mM} \mathrm{NaH}_{2} \mathrm{PO}_{4}$

$300 \mathrm{mM} \mathrm{NaCl}$

$250 \mathrm{mM}$ Imidazole, $\mathrm{pH}-8.0$

4. Storage buffer $(50 \mathrm{ml})$

$25 \mathrm{mM}$ MOPSO, $\mathrm{pH}$ to 7.0

$15 \%[v / v]$ glycerol

5. Assay buffer $(10 \mathrm{ml})$

$25 \mathrm{mM}$ MOPSO, pH to 7.0

$10 \%[v / v]$ glycerol

2 mM DTT

$10 \mathrm{mM} \mathrm{MgCl} 2$

6. Dephosphorylation buffer (2 $\mathrm{ml})$

$0.2 \mathrm{M}$ Tris- $\mathrm{HCl}, \mathrm{pH}-9.5$

2 units of CIP (stock 18 units $/ \mathrm{mg}$ )

2 units of potato apyrase (stock 25.2 units $/ \mathrm{mg}$ )

7. Mobile phase solvent $(50 \mathrm{ml})$

Methanol:water [95:5 v/v]

8. TG buffer

$25 \mathrm{mM}$ Tris

$192 \mathrm{mM}$ Glycine

$0.1 \%$ SDS 


\section{Acknowledgments}

This protocol was developed by modifying methods from Orlova et al. (2009) and Rai et al. (2013). This work was supported by the Department of Biotechnology supported projects BT/HRD/35/24/2006 and BT/PR6109/AGII/106/857/2012 to DAN. AR was supported by a Research Fellowship of University Grants Commission, New Delhi, India. The institutional communication number for this article is CIMAP/PUB/2020/APR/21.

\section{Competing interests}

The authors declare no competing interests.

\section{References}

1. Bradford, M. M. (1976). A rapid and sensitive method for the quantitation of microgram quantities of protein utilizing the principle of protein-dye binding. Anal Biochem 72: 248-254.

2. Kumar, S. R., Rai, A., Bomzan, D. P., Kumar, K., Hemmerlin, A., Dwivedi, V., Godbole, R. C., Barvkar, V., Shanker, K., Shilpashree, H. B., Bhattacharya, A., Smitha, A. R., Hegde, N. and Nagegowda, D. A. (2020). A plastid-localized bona fide geranylgeranyl diphosphate synthase plays a necessary role in monoterpene indole alkaloid biosynthesis in Catharanthus roseus. Plant J 103(1): 248-265.

3. Nagel, R., Gershenzon, J. and Schmidt, A. (2012). Nonradioactive assay for detecting isoprenyl diphosphate synthase activity in crude plant extracts using liquid chromatography coupled with tandem mass spectrometry. Anal Biochem 422(1): 33-38.

4. Orlova, I., Nagegowda, D. A., Kish, C. M., Gutensohn, M., Maeda, H., Varbanova, M., Fridman, E., Yamaguchi, S., Hanada, A., Kamiya, Y., Krichevsky, A., Citovsky, V., Pichersky, E. and Dudareva, N. (2009). The small subunit of snapdragon geranyl diphosphate synthase modifies the chain length specificity of tobacco geranylgeranyl diphosphate synthase in planta. Plant Cell 21(12): 4002-4017.

5. Rai, A., Smita, S. S., Singh, A. K., Shanker, K. and Nagegowda, D. A. (2013). Heteromeric and homomeric geranyl diphosphate synthases from Catharanthus roseus and their role in monoterpene indole alkaloid biosynthesis. Mol Plant 6(5): 1531-1549. 